\title{
On the Existence and Uniqueness of Solutions for Nonlinear System Modeling Three-Dimensional Viscous Stratified Flows
}

\author{
Andrei Giniatoulline, Tovias Castro \\ Department of Mathematics, Los Andes University, Bogota, Colombia \\ Email: aginiato@uniandes.edu.co, te.castro37@uniandes.edu.co
}

Received 1 March 2014; revised 1 April 2014; accepted 8 April 2014

Copyright (C) 2014 by authors and Scientific Research Publishing Inc.

This work is licensed under the Creative Commons Attribution International License (CC BY).

http://creativecommons.org/licenses/by/4.0/

\begin{abstract}
We establish the uniqueness and local existence of weak solutions for a system of partial differential equations which describes non-linear motions of viscous stratified fluid in a homogeneous gravity field. Due to the presence of the stratification equation for the density, the model and the problem are new and thus different from the classical Navier-Stokes equations.
\end{abstract}

\section{Keywords}

Partial Differential Equations, Sobolev Spaces, Fluid Dynamics, Stratified Fluid, Viscous Fluid

\section{Introduction}

The objective of this paper is to study the qualitative properties of the weak solutions of the system of partial differential equations which describes nonlinear motions of stratified three-dimensional viscous fluid in the gravity field, such as existence, uniqueness and smoothness. This model of three-dimensional stratified fluid corresponds to a stationary distribution of the initial density in a homogeneous gravitational field, which is of Boltzmann type and is exponentially decreasing with the growth of the altitude. The results may be applied in the mathematical fluid dynamics modelling real non-linear flows in the Atmosphere and the Ocean.

The additional unknown function (density), as well as the stratification equation itself, constitutes the novelty of the problem. To construct the solutions, we will use the Galerkin method.

We consider a bounded domain $\Omega \subset R^{3}$ with the boundary of the class $\partial \Omega \in C^{\infty}$ piecewise, and the following system of fluid dynamics

How to cite this paper: Giniatoulline, A. and Castro, T. (2014) On the Existence and Uniqueness of Solutions for Nonlinear System Modeling Three-Dimensional Viscous Stratified Flows. Journal of Applied Mathematics and Physics, 2, 528-539. 


$$
\left\{\begin{array}{l}
\frac{\partial u_{1}}{\partial t}-v \Delta u_{1}+\boldsymbol{u} \cdot \nabla u_{1}+\frac{\partial p}{\partial x_{1}}=0 \\
\frac{\partial u_{2}}{\partial t}-v \Delta u_{2}+\boldsymbol{u} \cdot \nabla u_{2}+\frac{\partial p}{\partial x_{2}}=0 \\
\frac{\partial u_{3}}{\partial t}-v \Delta u_{3}+\boldsymbol{u} \cdot \nabla u_{3}+g \rho+\frac{\partial p}{\partial x_{3}}=0 \\
\frac{\partial \rho}{\partial t}-\frac{N^{2}}{g} u_{3}=0 \\
\frac{\partial u_{1}}{\partial x_{1}}+\frac{\partial u_{2}}{\partial x_{2}}+\frac{\partial u_{3}}{\partial x_{3}}=0
\end{array}\right.
$$

Here $x=\left(x_{1}, x_{2}, x_{3}\right)$ is the space variable, $\boldsymbol{u}(x, t)=\left(u_{1}(x, t), u_{2}(x, t), u_{3}(x, t)\right)$ is the velocity field, $p(x, t)$ is the scalar field of the dynamic pressure and $p(x, t)$ is the dynamic density. In this model, the stationary distribution of density is described by the function $\mathrm{e}^{-N x_{3}}$, where $N$ is a positive constant. The gravitational constant $g$ and the viscosity coefficient $v$ are also assumed as strictly positive.

For linear non-viscous case, Equations (1.1) are deduced, for example, in [1]-[3]. For linear viscous compressible fluid, system 1.1 is deduced, for example, in [4]. The linear system corresponding to 1.1, was studied from various points of view, and some results may be found in [5]-[10].

There exists, of course, huge bibliography concerning Navier-Stokes system (see, for example, [11]-[14]).

However, the non-linear system modelling stratified viscous flows have not been studied mathematically yet, and thus our research was motivated by the novelty of the presence of the term $\rho$ in the third equation of 1.1, and also by the presence of the fourth equation itself.

\section{Construction and Existence of a Weak Solution}

We denote $e_{3}=(0,0,1)$ and observe that, without loss of generality we can consider $g=1$. In this way, we write Equations (1.1) in the vector form:

$$
\left\{\begin{array}{l}
\frac{\partial u}{\partial t}-v \Delta u+(u \cdot \nabla) u+\rho e_{3}+\nabla p=0 \\
\frac{\partial \rho}{\partial t}-N^{2} u \cdot e_{3}=0 \\
\operatorname{div} u=0
\end{array}\right.
$$

We associate system 1.2 with the initial conditions

and the Dirichlet boundary conditions

$$
\left\{\begin{array}{l}
u(x, 0)=u_{0}(x) \\
\rho(x, 0)=\rho_{0}(x)
\end{array}\right.
$$

$$
\left\{\begin{array}{l}
\left.u\right|_{\partial \Omega}=0 \\
\left.\rho\right|_{\partial \Omega}=0
\end{array} .\right.
$$

Let $J_{0}^{\infty}(\Omega)$ be a functional space of smooth solenoidal functions with compact support in $\Omega$. We denote as $J(\Omega)$ the closure of $J_{0}^{\infty}(\Omega)$ in the norm $L_{2}(\Omega)$. We also denote as $J_{1}(\Omega)$ the space of solenoidal functions from $C^{1}(\Omega)$ which satisfy homogeneous Dirichlet condition.

\section{Definition 1.}

Let $0<T<\infty, u(t, x) \in\left[L_{\infty}(0, T) \times J(\Omega)\right] \cap\left[L_{2}(0, T) \times J_{1}(\Omega)\right], \quad \rho(t, x) \in L_{\infty}(0, T) \times L_{2}(\Omega)$.

For any pair of vector functions $f, g \in L_{2}(\Omega)$ we denote $\langle f, g\rangle=\left(\int_{\Omega}\left(f_{1} g_{1}+f_{2} g_{2}+f_{3} g_{3}\right) \mathrm{d} x\right)^{1 / 2}$. 
We will call $(u, \rho)$ a weak solution of 1.2-1.4, if the following integral identities hold

$$
\begin{gathered}
\int_{0}^{T}\left\{\left\langle u, \Phi_{t}\right\rangle+\langle u,(u \cdot \nabla) \Phi\rangle+v\langle\nabla u, \nabla \Phi\rangle+\left\langle\rho e_{3}, \Phi\right\rangle\right\} \mathrm{d} t+\left\langle u_{0}, \Phi(0)\right\rangle=0 \\
\int_{0}^{T}\left\{\left(\rho, \Psi_{t}\right)_{L_{2}}+N^{2}\left(u e_{3}, \Psi\right)_{L_{2}}\right\} \mathrm{d} t+\left(\rho_{0}, \Psi(0)\right)_{L_{2}}=0,
\end{gathered}
$$

for every pair of functions $\Phi, \Psi$ such that

$$
\Phi \in C^{1}(0, T) \times J_{1}(\Omega), \quad \Psi \in C^{1}(0, T) \times L_{2}(\Omega) \text {, and } \Phi(T)=0, \Psi(T)=0 .
$$

We observe that the relations 1.5 - 1.6 are obtained in a natural way after multiplying by $(\Phi, \Psi)$ the system 1.2, and integrating by parts over $\Omega$ for $x$ and over the interval $(0, T)$ for $t$. After knowing the functions $(u, \rho)$, the function $\nabla p$ can be easily found from 1.2.

Let $\left\{\varphi_{n}\right\}$ be a complete orthonormal system in $J(\Omega)$. We observe that, without loss of generality, it can be chosen as a system of eigenfunctions of Stokes operator (see, for example, [11] [13] [15]).

We construct the solutions of 1.2 as Galerkin approximations

$$
u^{n}(t, x)=\sum_{k=1}^{n} c_{k n}(t) \varphi_{k}(x),
$$

where $c_{n k}(t) \in C^{1}[0, T]$ are unknown coefficients and $\rho_{n}(t, x)$ are obtained as solutions of Cauchy problem

$$
\rho_{t}^{n}(t, x)=N^{2} u^{n} e_{3}, \rho^{n}(0, x)=\rho_{0}(x) .
$$

Our primary aim is to determine the existence of $c_{k n}(t)$ and the resulting properties of the approximations $u^{n}$.

If we consider 1.5 for $u^{n}$ and $\Phi(t, x)=H(t) \varphi_{k}(x), H \in C^{1}(0, T), H(T)=0$, then, the arbitrary election of $H(t)$ will imply the relations

$$
\begin{aligned}
& \left\langle u_{t}^{n}-v \Delta u^{n}+\left(u^{n} \cdot \nabla\right) u^{n}+\rho^{n} e_{3}, \varphi_{k}\right\rangle=0, \\
& k=1, \cdots, n .
\end{aligned}
$$

We transform now the system 1.9 into an autonomous system of differential equations of the first order with respect to the variables $c_{k n}$, where the vector field is $C^{\infty}$. If we denote $x(t)=\left(c_{1 n}(t), \cdots, c_{n n}(t)\right), \beta_{j i}^{k}=\left\langle\left(\varphi_{j} \cdot \nabla\right) \varphi_{i}, \varphi_{k}\right\rangle$, then it can be easily seen that the system 1.9 is equivalent to

$$
x^{\prime}(t)=F(x(t))+N^{2} \int_{0}^{t} G x(s) \mathrm{d} s
$$

where

$$
\begin{gathered}
F(x)=\left(\begin{array}{c}
x^{\mathrm{T}} M_{1} x \\
\vdots \\
x^{\mathrm{T}} M_{n} x
\end{array}\right)+v H x+\left(\begin{array}{c}
\left\langle\rho_{0} e_{3}, \varphi_{1}\right\rangle \\
\vdots \\
\left\langle\rho_{0} e_{3}, \varphi_{n}\right\rangle
\end{array}\right), \\
M_{k}=\left(\begin{array}{ccc}
\beta_{11}^{k} & \cdots & \beta_{1 n}^{k} \\
\vdots & \ddots & \vdots \\
\beta_{n 1}^{k} & \cdots & \beta_{n n}^{k}
\end{array}\right), G=\left(\begin{array}{ccc}
\left\langle\left(\varphi_{1} \cdot e_{3}\right) e_{3}, \varphi_{1}\right\rangle & \cdots & \left\langle\left(\varphi_{n} \cdot e_{3}\right) e_{3}, \varphi_{1}\right\rangle \\
\vdots & \ddots & \vdots \\
\left\langle\left(\varphi_{1} \cdot e_{3}\right) e_{3}, \varphi_{n}\right\rangle & \cdots & \left\langle\left(\varphi_{n} \cdot e_{3}\right) e_{3}, \varphi_{n}\right\rangle
\end{array}\right), \\
H=\left(\begin{array}{ccc}
\left\langle\nabla \varphi_{1}, \nabla \varphi_{1}\right\rangle & \cdots & \left\langle\nabla \varphi_{n}, \nabla \varphi_{1}\right\rangle \\
\vdots & \ddots & \vdots \\
\left\langle\nabla \varphi_{1}, \nabla \varphi_{n}\right\rangle & \cdots & \left\langle\nabla \varphi_{n}, \nabla \varphi_{n}\right\rangle
\end{array}\right),\langle\nabla \Phi, \nabla \Psi\rangle=\sum_{i=1}^{3}\left\langle\nabla \Phi_{i}, \nabla \Psi_{i}\right\rangle .
\end{gathered}
$$


After differentiating 1.10, we obtain

$$
x^{\prime \prime}(t)=K\left(x, x^{\prime}\right),
$$

where $K_{j}\left(x, x^{\prime}\right)=M_{j} x^{\prime} \cdot x+M_{j} x \cdot x^{\prime}+v\left(H \cdot x^{\prime}\right)_{j}+N^{2}(G \cdot x)_{j}, j=1, \cdots, n$.

We introduce the notation $x^{\prime}=z$ and thus rewrite 1.11 as

$$
\left(\begin{array}{l}
x \\
z
\end{array}\right)^{\prime}=L(x, z)
$$

Since $L(x, z)$ is an infinitely differentiable vector field, then, from the theory of ordinary differential equations, we conclude that 1.12 admits a maximal solution in the interval $\left[0, T_{n}\right]$.

Now, we shall deduce some estimates to prove that $T_{n}=T$ is independent on $n$.

\section{Lemma 1.}

The solutions $u^{n}(t, x)$ of the approximate system 1.9 are defined uniquely by 1.7.

Additionally, the following estimates are valid. For all $n \in N$ there exists $T^{*}>0$

such that

1) $\sup _{0 \leq t \leq T^{*}}\left\|u^{n}(t, x)\right\|^{2} \leq\left\|u_{0}\right\|^{2}+\frac{1}{N^{2}}\left\|\rho_{0}\right\|^{2}$,

2) $\int_{0}^{t}\left\|\nabla u^{n}(\tau, x)\right\|^{2} \mathrm{~d} \tau \leq \frac{1}{v}\left(\left\|u_{0}\right\|^{2}+\frac{1}{N^{2}}\left\|\rho_{0}\right\|^{2}\right)$ for all $t \in\left[0, T^{*}\right]$,

3) $\left\|u_{t}^{n}\right\|^{2} \leq \sqrt{\frac{a}{b}} \tan \left(\sqrt{a b} T^{*}+M\right)$ for all $t \in\left[0, T^{*}\right]$,

4) $\left\|\nabla u^{n}(t, x)\right\|^{2} \leq \frac{2}{v}\left(\left\|u_{0}\right\|+\frac{1}{N}\left\|\rho_{0}\right\|\right)^{2}+\frac{1}{2 v} \sqrt{\frac{a}{b}} \tan \left(\sqrt{a b} T^{*}+M\right)$ for all $t \in\left[0, T^{*}\right]$,

5) $\int_{0}^{t}\left\|u_{t}^{n}(\tau, x)\right\|_{L_{2}}^{2} \mathrm{~d} \tau \leq \frac{1}{b} \ln \left|\frac{\sec (\sqrt{a b} t+M)}{\sec M}\right|$ for all $t \in\left[0, T^{*}\right]$,

6) $\int_{0}^{t}\left\|u^{n}(\tau, x)\right\|_{W_{2}^{1}}^{2} \mathrm{~d} \tau \leq\left(\left\|u_{0}\right\|^{2}+\frac{1}{N^{2}}\left\|\rho_{0}\right\|^{2}\right)\left(t+\frac{1}{v}\right)$ for all $t \in\left[0, T^{*}\right]$,

where the positive constants $a, b, M$ and $T^{*}$ depend only on the initial data 1.3, the parameter of the system $\mathrm{N}$ and the domain $\Omega$.

Remark.

The values of the constants $a, b, M$ and $T^{*}$ are given below in 1.22, 1.28 and 1.29.

Proof.

For $c>0$ we introduce the following auxiliary real-valued function $E(t)=\frac{1}{2}\left\|u^{n}\right\|^{2}+\frac{c}{2}\left\|\rho^{n}\right\|^{2}$.

We observe that

$$
E^{\prime}(t)=\left\langle u_{t}^{n}, u^{n}\right\rangle+c\left(\rho_{t}^{n}, \rho\right)_{L_{2}}=\left\langle u_{t}^{n}, u^{n}\right\rangle+c N^{2}\left(u_{3}^{n}, \rho\right)_{L_{2}} .
$$

After multiplying each equation of 1.9 by $c_{n k}(t)$ and summing them with respect to $k$, we obtain

$$
\left\langle u_{t}^{n}, u^{n}\right\rangle+v\left\|\nabla u^{n}\right\|^{2}+\left\langle\rho^{n} e_{3}, u^{n}\right\rangle=0 .
$$

In this way, choosing $c=\frac{1}{N^{2}}$, we have that

$$
E^{\prime}(t)=-v\left\|\nabla u^{n}\right\|^{2} \leq 0 .
$$

Therefore, $E(t) \leq E(0)$ for all $t$, and thus 


$$
\left\|u^{n}\right\|^{2}+\frac{1}{N^{2}}\left\|\rho^{n}\right\|^{2} \leq\left\|u_{0}\right\|^{2}+\frac{1}{N^{2}}\left\|\rho_{0}\right\|^{2} .
$$

In particular, we have $\sum_{j=1}^{n}\left|c_{j n}(t)\right|^{2}=\left\|u^{n}\right\|^{2} \leq\left\|u_{0}\right\|^{2}+\frac{1}{N^{2}}\left\|\rho_{0}\right\|^{2}$. Thus the statement "a)" of the Lemma is proved.

From 1.14 we obtain that

$$
\int_{0}^{t}\left\|\nabla u^{n}(\tau, x)\right\|^{2} \mathrm{~d} \tau \leq \frac{1}{v}\left(\left\|u_{0}\right\|^{2}+\frac{1}{N^{2}}\left\|\rho_{0}\right\|^{2}\right)
$$

which will prove the statement "b)”. Indeed,

$$
\int_{0}^{t} v\left\|\nabla u^{n}(\tau, x)\right\|^{2} \mathrm{~d} \tau=-\int_{0}^{t} E^{\prime}(\tau) \mathrm{d} \tau=E(0)-E(t) \leq E(0)+E(t) \leq 2 E(0)=\left\|u_{0}\right\|^{2}+\frac{1}{N^{2}}\left\|\rho_{0}\right\|^{2} .
$$

Now, differentiating 1.9, we obtain $\left\langle u_{t t}^{n}-v \Delta u_{t}^{n}+\left(u_{t}^{n} \cdot \nabla\right) u^{n}+\left(u^{n} \cdot \nabla\right) u_{t}^{n}+\rho_{t}^{n} e_{3}, \varphi_{k}\right\rangle=0$.

We multiply the last relations by $c_{k n}^{\prime}(t)$ and sum them with respect to $k$ :

$$
\left\langle u_{t t}^{n}, u_{t}^{n}\right\rangle+v\left\|\nabla u_{t}^{n}\right\|^{2}+\left\langle\left(u_{t}^{n} \cdot \nabla\right) u^{n}, u_{t}^{n}\right\rangle+\left\langle\left(u^{n} \cdot \nabla\right) u_{t}^{n}, u_{t}^{n}\right\rangle+\left\langle\rho_{t}^{n} e_{3}, u_{t}^{n}\right\rangle=0 .
$$

Therefore, keeping in mind that $\left\langle\left(u^{n} \cdot \nabla\right) u_{t}^{n}, u_{t}^{n}\right\rangle=0$, we have

$$
\frac{1}{2} \frac{\mathrm{d}}{\mathrm{d} t}\left\|u_{t}^{n}\right\|^{2}+v\left\|\nabla u_{t}^{n}\right\|^{2}=-\left\langle\left(u_{t}^{n} \cdot \nabla\right) u^{n}, u_{t}^{n}\right\rangle-N^{2}\left\langle u_{3}^{n} e_{3}, u_{t}^{n}\right\rangle .
$$

We would like to estimate the right-hand terms in 1.17. Evidently, from 1.15 we obtain

$$
\mid N^{2}\left\langle u_{3}^{n} e_{3}, u_{t}^{n}\right\rangle \leq N^{2}\left\|u^{n}\right\|\left\|u_{t}^{n}\right\| \leq \frac{N^{2}}{2}\left(\left\|u^{n}\right\|^{2}+\left\|u_{t}^{n}\right\|^{2}\right) \leq \frac{N^{2}}{2}\left(\left\|u_{0}\right\|^{2}+\frac{1}{N^{2}}\left\|\rho_{0}\right\|^{2}\right)+\frac{N^{2}}{2}\left\|u_{t}^{n}\right\|^{2} .
$$

We will need the following estimate:

$$
\begin{aligned}
v\left\|\nabla u^{n}\right\|^{2} & =-\left\langle u_{t}^{n}, u^{n}\right\rangle-\left\langle\rho^{n} e_{3}, u^{n}\right\rangle \leq\left|\left\langle u_{t}^{n}, u^{n}\right\rangle\right|+\left|\left\langle\rho^{n} e_{3}, u^{n}\right\rangle\right| \\
& \leq\left\|u^{n}\right\|\left\|u_{t}^{n}\right\|+\left\|u^{n}\right\|\left\|\rho^{n}\right\| \leq\left(\left\|u_{0}\right\|+\frac{1}{N}\left\|\rho_{0}\right\|\right)\left\|u_{t}^{n}\right\|+\left(\left\|u_{0}\right\|+\frac{1}{N}\left\|\rho_{0}\right\|\right)^{2} .
\end{aligned}
$$

On the other hand, from the generalized Hölder inequality and the interpolation inequality $\|f\|_{L_{4}} \leq\|f\|_{L_{2}}^{\frac{1}{4}}\|f\|_{L_{6}}^{\frac{3}{4}}$, we can estimate the term

$$
\left\langle\left(u_{t}^{n} \cdot \nabla\right) u^{n}, u_{t}^{n}\right\rangle \leq\left\|u_{t}^{n}\right\|_{L_{4}}\left\|\nabla u^{n}\right\|_{L_{2}}\left\|u_{t}^{n}\right\|_{L_{4}}=\left\|u_{t}^{n}\right\|_{L_{4}}^{2}\left\|\nabla u^{n}\right\|_{L_{2}} \leq\left\|u_{t}^{n}\right\|_{L_{2}}^{\frac{1}{2}}\left\|u_{t}^{n}\right\|_{L_{6}}^{\frac{3}{2}}\left\|\nabla u^{n}\right\|_{L_{2}} .
$$

From the Young inequality with " $\varepsilon$ ” for $p=4, \quad q=\frac{3}{4}, \quad x=\left\|u_{t}^{n}\right\|_{L_{2}}^{\frac{1}{2}}\left\|\nabla u^{n}\right\|_{L_{2}}, y=\left\|u_{t}^{n}\right\|_{L_{2}}^{\frac{1}{2}}$, we obtain

$$
\left|\left\langle\left(u_{t}^{n} \cdot \nabla\right) u^{n}, u_{t}^{n}\right\rangle\right| \leq \varepsilon\left\|\nabla u^{n}\right\|_{L_{2}}^{4}\left\|u_{t}^{n}\right\|_{L_{2}}^{2}+C(\varepsilon)\left\|u_{t}^{n}\right\|_{L_{6}}^{2}
$$

Now, using 1.19 and the inclusion Sobolev inequality $\left\|u_{t}^{n}\right\|_{L_{6}}^{2} \leq C^{\prime}\left\|\nabla u_{t}^{n}\right\|_{L_{2}}^{2}$, we have

$$
\left|\left\langle\left(u_{t}^{n} \cdot \nabla\right) u^{n}, u_{t}^{n}\right\rangle\right| \leq \frac{2 \varepsilon}{v}\left(\left(\left\|u_{0}\right\|+\frac{1}{N}\left\|\rho_{0}\right\|\right)^{4}\left\|u_{t}^{n}\right\|_{L_{2}}^{2}+\left(\left\|u_{0}\right\|+\frac{1}{N}\left\|\rho_{0}\right\|\right)^{2}\left\|u_{t}^{n}\right\|_{L_{2}}^{4}\right)+C(\varepsilon) C^{\prime}\left\|\nabla u_{t}^{n}\right\|_{L_{2}}^{2} .
$$

If we choose $\varepsilon$ such that $C(\varepsilon) C^{\prime}=\frac{v}{2}$, then the last estimate, together with 1.17 and 1.18, implies 


$$
\frac{1}{2} \frac{\mathrm{d}}{\mathrm{d} t}\left\|u_{t}^{n}\right\|^{2} \leq a+b\left\|u_{t}^{n}\right\|_{L_{2}}^{4}, a=\frac{\left(\frac{1}{N^{2}}+\frac{2 \varepsilon}{v}\left(\left\|u_{0}\right\|+\frac{1}{N}\left\|\rho_{0}\right\|\right)^{4}\right)^{2}}{\frac{8 \varepsilon}{v}\left(\left\|u_{0}\right\|+\frac{1}{N}\left\|\rho_{0}\right\|\right)^{2}}, b=\frac{4 \varepsilon}{v}\left(\left\|u_{0}\right\|+\frac{1}{N}\left\|\rho_{0}\right\|\right)^{2} .
$$

Evidently, from 1.22 we have

$$
\frac{1}{2} \int_{0}^{t} \frac{\frac{\mathrm{d}}{\mathrm{d} t}\left\|u_{t}^{n}\right\|^{2}}{a+b\left\|u_{t}^{n}\right\|^{4}} \leq t,
$$

from which, after integrating with respect to $t$, it follows that

$$
\left\|u_{t}^{n}\right\|^{2} \leq \sqrt{\frac{a}{b}} \tan \left(\sqrt{a b} t+\tan ^{-1}\left(\frac{\sqrt{b}\left\|u_{t}^{n}(0, x)\right\|^{2}}{\sqrt{a}}\right)\right) .
$$

Now, to obtain a uniform upper estimate with respect to $n$ for $\left\|u_{t}^{n}\right\|^{2}$, we only need to prove such estimate for $\left\|u_{t}^{n}(0, x)\right\|^{2}$. We remind that the system $\left\{\varphi_{k}\right\}$, being formed by eigenfunctions of Stokes operator is orthonor mal in $J(\Omega)$, and also in $J_{1}(\Omega)$ with the scalar product $(\nabla \Phi, \nabla \Psi)$, as well as in $J_{1}(\Omega) \cap W_{2}^{2}(\Omega)$ with the scalar product $(P \nabla \Phi, P \nabla \Psi)$, where $P$ is an orthogonal projection of $L_{2}(\Omega)$ onto $J(\Omega)$. From 1.9 we have

$$
\left\|u_{t}^{n}(0)\right\|^{2}=-\left\langle\rho^{n}(0) e_{3}, u_{t}^{n}(0)\right\rangle-v\left\langle P \Delta u_{t}^{n}(0), u_{t}^{n}(0)\right\rangle-\left\langle\left(u^{n}(0) \cdot \nabla\right) u^{n}(0), u_{t}^{n}(0)\right\rangle .
$$

Proceeding analogously to 1.17-1.21 and also using Lemma 1 from [11], we obtain

$$
\begin{aligned}
\left\|u_{t}^{n}(0)\right\| & \leq\|\rho(0)\|+v\left\|P \Delta u^{n}(0)\right\|+\left\|u^{n}(0) \nabla u^{n}(0)\right\| \leq\|\rho(0)\|+v\left\|P \Delta u^{n}(0)\right\|+\left\|u^{n}(0)\right\|_{L_{6}}\left\|\nabla u^{n}(0)\right\|_{L_{3}} \\
& \leq\|\rho(0)\|+v\left\|P \Delta u^{n}(0)\right\|+C^{\prime}\left\|\nabla u^{n}(0)\right\|_{L_{2}}\left(\left\|P \Delta u^{n}(0)\right\|_{L_{2}}^{\frac{1}{2}}\left\|\nabla u^{n}(0)\right\|_{L_{2}}^{\frac{1}{2}}+\left\|\nabla u^{n}(0)\right\|_{L_{2}}\right) \\
& \leq\|\rho(0)\|+v\left\|P \Delta u^{n}(0)\right\|+C^{\prime}\left\|P \nabla u^{n}(0)\right\|_{L_{2}}^{\frac{1}{2}}\left\|\nabla u^{n}(0)\right\|_{L_{2}}^{\frac{3}{2}}+C^{\prime}\left\|\nabla u^{n}(0)\right\|_{L_{2}}^{2} \\
& \leq\|\rho(0)\|+\frac{v^{2}}{2}+\frac{1}{2}\left\|P \Delta u^{n}(0)\right\|^{2}+\left(C^{\prime}\right)^{4} \varepsilon\left\|P \nabla u^{n}(0)\right\|_{L_{2}}^{2}+C(\varepsilon)\left\|\nabla u^{n}(0)\right\|_{L_{2}}^{2}+C^{\prime}\left\|\nabla u^{n}(0)\right\|_{L_{2}}^{2} .
\end{aligned}
$$

If we choose $\varepsilon=1 / 2\left(C^{\prime}\right)^{4}$, then we finally have

$$
\left\|u_{t}^{n}(0)\right\| \leq\|\rho(0)\|+\frac{v^{2}}{2}+\left\|P \Delta u^{n}(0)\right\|^{2}+\left(C^{\prime}+C(\varepsilon)\right)\left\|\nabla u^{n}(0)\right\|^{2} .
$$

Now, from the Bessel inequality and the properties

$$
c_{k n}(0)=\left\langle\varphi_{k}, u(0)\right\rangle=\frac{1}{\left\|\nabla \varphi_{k}\right\|^{2}}\left\langle\nabla \varphi_{k}, \nabla u(0)\right\rangle=\frac{1}{\left\|P \Delta \varphi_{k}\right\|^{2}}\left\langle P \Delta \varphi_{k}, P \Delta u(0)\right\rangle,
$$

we can express 1.25 as

$$
\left\|u_{t}^{n}(0)\right\| \leq\|\rho(0)\|+\frac{v^{2}}{2}+\|P \Delta u(0)\|^{2}+\left(C^{\prime}+C(\varepsilon)\right)\|\nabla u(0)\|^{2} .
$$

In this way, using 1.27 and the evident inequality $(x+y+z)^{2} \leq 4\left(x^{2}+y^{2}+z^{2}\right)$, we can estimate 1.24 as

$$
\left\|u_{t}^{n}\right\|^{2} \leq \sqrt{\frac{a}{b}} \tan (\sqrt{a b} t+M)
$$


where $M=\tan ^{-1}\left(\frac{4 \sqrt{b}\left(\left(\|\rho(0)\|+\frac{v^{2}}{2}\right)^{2}+\|P \Delta u(0)\|^{4}+\left(C(\varepsilon)+C^{\prime}\right)^{2}\|\nabla u(0)\|^{4}\right)}{a}\right)$.

Taking $0<T^{*}<\frac{\frac{\pi}{2}-M}{\sqrt{a b}}$, we assure that $\tan \left(\sqrt{a b} T^{*}+M\right)<\infty$ and thus we obtain the result that for all $n \in N$ there exists $T^{*}>0$ such that

$$
\left\|u_{t}^{n}\right\|^{2} \leq \sqrt{\frac{a}{b}} \tan \left(\sqrt{a b} T^{*}+M\right) \text { for all } t \in\left[0, T^{*}\right],
$$

which proves the statement "c)" of the Lemma. It is easy to see that the statement "d)" is a direct consequence of 1.19 and 1.29. The statement "e)" of the Lemma is obtained immediately if we integrate both sides of 1.29 with respect to $t$. It remains to prove the statement "f)". For that, we use 1.15 and 1.16 and therefore obtain

$$
\int_{0}^{t}\left\|u^{n}(\tau, x)\right\|_{W_{2}^{1}}^{2} \mathrm{~d} \tau=\int_{0}^{t}\left(\left\|u^{n}(\tau, x)\right\|^{2}+\left\|\nabla u^{n}(\tau, x)\right\|^{2}\right) \mathrm{d} \tau \leq\left(\left\|u_{0}\right\|^{2}+\frac{1}{N^{2}}\left\|\rho_{0}\right\|^{2}\right)\left(t+\frac{1}{v}\right),
$$

which concludes the proof of the Lemma.

We would like to obtain now more estimates for the approximate solutions $u^{n}(t, x)$, with an intention to show that their limit, which is an obvious candidate for a solution of $1.2-1.4$, would preserve certain regularity properties of $u^{n}$.

Lemma 2.

For all $n \in N$ and for all $t \in\left[0, T^{*}\right]$ there exists a constant $C>0$ which does not depend on $u^{n}$, such that the following estimate is valid:

$$
\int_{0}^{t}\left\|u^{n}(\tau, x)\right\|_{W_{2}^{2}}^{2} \mathrm{~d} \tau \leq C\left(T^{*},\left\|u_{0}\right\|,\left\|\rho_{0}\right\|, N, \Omega\right)
$$

Proof.

We continue using the notation of $P$ as an orthogonal projection of $L_{2}(\Omega)$ onto $J(\Omega)$. Let $\lambda_{k}$ be eigenvalues corresponding to the eigenfunctions $\varphi_{k}$ of the Stokes operator. We multiply 1.9 by $c_{k n} \lambda_{k}$ and sum up the resulting equations with respect to $k$. In this way, we have

$$
\left\langle u_{t}^{n}-v \Delta u^{n}+\left(u^{n} \cdot \nabla\right) u^{n}+\rho^{n} e_{3},-P \Delta \varphi_{k}\right\rangle=0 .
$$

We would like to estimate the following term in 1.31:

$$
\left\langle v \Delta u^{n}, P \Delta \varphi_{k}\right\rangle=\left\langle u_{t}^{n}, P \Delta \varphi_{k}\right\rangle+\left\langle\left(u^{n} \cdot \nabla\right) u^{n}, P \Delta \varphi_{k}\right\rangle+\left\langle\rho^{n} e_{3}, P \Delta \varphi_{k}\right\rangle .
$$

By using Cauchy and Hölder inequality, together with the inequality $x y \leq \varepsilon x^{2}+\frac{1}{4 \varepsilon} y^{2}$, we obtain

$$
\left\langle v \Delta u^{n}, P \Delta \varphi_{k}\right\rangle \leq \varepsilon\left\{\left\|u_{t}^{n}\right\|^{2}+\left\|u^{n}\right\|_{L_{6}}^{2}\left\|\nabla u^{n}\right\|_{L_{3}}^{2}+\left\|\rho^{n} e_{3}\right\|^{2}\right\}+\frac{1}{4 \varepsilon}\left\|P \Delta \varphi_{k}\right\|^{2} .
$$

Now we use Sobolev inclusion inequality and Lemma 1 from [11], which allows us to estimate 1.33 as

$$
\left\langle v \Delta u^{n}, P \Delta \varphi_{k}\right\rangle \leq \varepsilon\left\{\left\|u_{t}^{n}\right\|^{2}+C^{\prime}\left\|u^{n}\right\|_{L_{2}}^{2}\left(\left\|P \nabla u^{n}\right\|^{\frac{1}{2}}\left\|\nabla u^{n}\right\|^{\frac{1}{2}}+\left\|\nabla u^{n}\right\|\right)^{2}+\left\|\rho^{n} e_{3}\right\|^{2}\right\}+\frac{3}{4 \varepsilon}\left\|P \Delta \varphi_{k}\right\|^{2} .
$$

We take $\varepsilon=\frac{3}{2 v}$, consider the inequality $(x+y)^{2} \leq 2\left(x^{2}+y^{2}\right)$ and use the property 


$$
\left\langle\Delta u^{n}, P \Delta \varphi_{k}\right\rangle=\left\langle\Delta u^{n}, P^{2} \Delta \varphi_{k}\right\rangle=\left\langle P \Delta u^{n}, P \Delta \varphi_{k}\right\rangle=\left\|P \Delta u^{n}\right\|^{2} .
$$

Proceeding in this way, we obtain

$$
\begin{aligned}
\frac{v}{2}\left\|P \Delta u^{n}\right\|^{2} & \leq \frac{3}{2 v}\left\{\left\|u_{t}^{n}\right\|^{2}+2 C^{\prime}\left\|\nabla u^{n}\right\|^{2}\left(\left\|P \Delta u^{n}\right\|\left\|\nabla u^{n}\right\|+\left\|\nabla u^{n}\right\|^{2}\right)+\left\|\rho^{n} e_{3}\right\|^{2}\right\} \\
& =\frac{3}{2 v}\left\{\left\|u_{t}^{n}\right\|^{2}+2 C^{\prime}\left\|\nabla u^{n}\right\|^{3}\left\|P \Delta u^{n}\right\|+2 C^{\prime}\left\|\nabla u^{n}\right\|^{4}+\left\|\rho^{n} e_{3}\right\|^{2}\right\} \\
& \leq \frac{3}{2 v}\left\{\left\|u_{t}^{n}\right\|^{2}+4 \eta C^{\prime 2}\left\|\nabla u^{n}\right\|^{6}+\frac{1}{4 \eta}\left\|P \Delta u^{n}\right\|^{2}+2 C^{\prime}\left\|\nabla u^{n}\right\|^{4}+\left\|\rho^{n} e_{3}\right\|^{2}\right\} .
\end{aligned}
$$

Now, if we take $\eta=\frac{3}{2 v^{2}}$, we will finally have

$$
\begin{aligned}
& \left\|P \Delta u^{n}\right\|^{2} \leq K\left\{\left\|u_{t}^{n}\right\|^{2}+\left\|\nabla u^{n}\right\|^{6}+\left\|\nabla u^{n}\right\|^{4}+\left\|\rho^{n} e_{3}\right\|^{2}\right\}, \\
& K=\frac{3}{v^{2}} \max \left\{1,2 C^{\prime}, \frac{6}{v^{2}} C^{\prime 2}\right\} .
\end{aligned}
$$

The term $\left\|u_{t}^{n}\right\|^{2}$ on the right side of 1.35 can be estimated by 1.29. The terms $\left\|\nabla u^{n}\right\|^{4}$ and $\left\|\nabla u^{n}\right\|^{6}$, can be estimated analogously, we use the statement “d)" of Lemma 1 and the inequality $(x+y)^{p} \leq 2^{p}\left(x^{p}+y^{p}\right)$, where we consider $p=2$ and $p=3$. Now, taking into account the properties

$$
\begin{gathered}
\int \tan ^{2}(\sqrt{a b} \tau+M) \mathrm{d} \tau=\frac{1}{\sqrt{a b}}[\tan (\sqrt{a b} \tau+M)-(\sqrt{a b} \tau+M)] \\
\int \tan ^{3}(\sqrt{a b} \tau+M) \mathrm{d} \tau=\frac{1}{\sqrt{a b}}\left[\frac{1}{2} \tan ^{2}(\sqrt{a b} \tau+M)+\ln |\cos (\sqrt{a b} \tau+M)|\right]
\end{gathered}
$$

we can proceed estimating the integral of 1.35 as follows:

$$
\begin{aligned}
& \int_{0}^{t}\left\|P \Delta u^{n}(\tau, x)\right\|^{2} \mathrm{~d} \tau \\
& \leq K\left\{\frac{1}{b} \ln \left|\frac{\sec (\sqrt{a b} t+M)}{\sec M}\right|+64\left(\left\|u_{0}\right\|+\frac{1}{N}\left\|\rho_{0}\right\|\right)^{6} t+\frac{a}{b^{2}}\left(\tan ^{2}(\sqrt{a b} t+M)-\frac{1}{2} \tan ^{2} M+\ln \left|\frac{\cos (\sqrt{a b} t+M)}{\cos M}\right|\right)\right. \\
& \left.+16\left(\left\|u_{0}\right\|+\frac{1}{N}\left\|\rho_{0}\right\|\right)^{4} t+\frac{1}{b} \sqrt{\frac{a}{b}}(\tan (\sqrt{a b} t+M)-M)+\left(\left\|u_{0}\right\|^{2}+\frac{1}{N^{2}}\left\|\rho_{0}\right\|^{2}\right) t\right\}
\end{aligned}
$$

We note that the estimate 1.38 is valid for $t \in\left[0, T^{*}\right]$. From $1.28-1.29$ we have that the value $T^{*}$ is chosen in such a way that the right side of 1.38 is positive. Finally, we use the result from [15] where there is shown that in the functional space $J_{1}(\Omega) \cap W_{2}^{2}(\Omega)$, the norms $\|P \Delta f\|_{L_{2}}$ and $\|f\|_{W_{2}^{2}}$ are equivalent, which concludes the proof.

Theorem 1.

Let $\Omega \subset R^{3}$ be a bounded domain with the boundary of the class $C^{\infty}$, and let $u_{0} \in J_{1}(\Omega) \cap W_{2}^{2}(\Omega)$, $\rho_{0} \in L_{2}(\Omega)$.

Then, there exists an interval $\left(0, T^{*}\right)$ and there exist the functions $u(t, x), \rho(t, x), p(t, x)$ which satisfy the system 1.2 and the conditions 1.3 - 1.4 in sense of $1.5-1.6$, such that 


$$
\begin{aligned}
& u \in\left[L_{\infty}\left(0, T^{*}\right) \times J(\Omega)\right] \cap\left[L_{2}\left(0, T^{*}\right) \times J_{1}(\Omega)\right], \\
& u \in\left[C_{0}\left[0, T^{*}\right] \times J_{1}(\Omega)\right] \cap\left[L_{2}\left(0, T^{*}\right) \times J_{1}(\Omega) \cap W_{2}^{2}(\Omega)\right], \\
& p \in L_{2}\left(0, T^{*}\right) \times W_{2}^{1}(\Omega), \\
& \rho \in L_{\infty}\left(0, T^{*}\right) \times L_{2}(\Omega), \\
& \frac{\partial u}{\partial t} \in L_{2}\left(0, T^{*}\right) \times J(\Omega) .
\end{aligned}
$$

Proof.

From Lemma 1 and Lemma 2 we have that there exist a function $u$ and a subsequence of $\left\{u^{n}\right\}$ (which, for brevity, we will denote also as $\left\{u^{n}\right\}$ ), such that

$$
\left\{\begin{array}{l}
u^{n} \rightarrow u \text { * weakly in } L_{\infty}\left(0, T^{*}\right) \times J(\Omega), \\
u^{n} \rightarrow u \text { weakly in } L_{2}\left(0, T^{*}\right) \times\left[J_{1}(\Omega) \cap W_{2}^{2}(\Omega)\right], \\
\frac{\partial u^{n}}{\partial t} \rightarrow \frac{\partial u}{\partial t} \text { weakly in } L_{2}\left(0, T^{*}\right) \times J(\Omega) .
\end{array}\right.
$$

We note that the inclusion $J_{1}(\Omega) \subset J(\Omega)$ is compact and continuous, and that $\left\{u^{n}\right\}$ is bounded in $L_{2}\left(0, T^{*}\right) \times J_{1}(\Omega)$ from Lemma 1 . On the other hand, from the statement "c)" of Lemma 1 we have that for $h>0$ and $0<t<T^{*}-h$, the following estimate holds:

$$
\int_{0}^{T^{*}-h}\left\|u^{n}(t+h)-u^{n}(t)\right\|^{2} \mathrm{~d} t \leq \int_{0}^{T^{*}-h t} \int_{t}^{h+h} \tan \left(\sqrt{a b} T^{*}+M\right) \mathrm{d} \tau \mathrm{d} t \leq h T^{*} \tan \left(\sqrt{a b} T^{*}+M\right) .
$$

In this way, from 1.39, 1.40 and Lemma 24.5 [12], it follows that $\left\{u^{n}\right\}$ belongs to a compact set in $L_{2}\left(0, T^{*}\right) \times J(\Omega)$. Therefore, the subsequence in 1.39 can be chosen in such a way that

$$
u^{n} \rightarrow u \text { strongly in } L_{2}\left(0, T^{*}\right) \times J(\Omega) .
$$

It is easy to see that $u$ satisfies the regularity properties of the Theorem. Indeed, since $u \in L_{2}\left(0, T^{*}\right) \times\left[J_{1}(\Omega) \cap W_{2}^{2}(\Omega)\right]$ and $u_{t} \in L_{2}\left(0, T^{*}\right) \times J(\Omega)$, then, from Theorem IV.5.11 [15] we obtain that $u \in C_{0}\left[0, T^{*}\right] \times J_{1}(\Omega)$. Using Sobolev inequality we have the estimate

$$
\left\|\left(u^{n} \cdot \nabla\right) u^{n}\right\|_{L_{2}}^{4} \leq C_{1}\left\|u^{n}\right\|_{L_{6}}^{4}\left\|\nabla u^{n}\right\|_{L_{3}}^{4} \leq C_{2}\left\|\nabla u^{n}\right\|_{L_{2}}^{4}\left(\left\|\nabla u^{n}\right\|_{L_{2}}^{\frac{1}{2}}\left\|u^{n}\right\|_{W_{2}^{2}}^{\frac{1}{2}}\right)^{4} \leq C_{3}\left\|\nabla u^{n}\right\|_{L_{2}}^{6}\left\|P \Delta u^{n}\right\|_{L_{2}}^{2} \leq C .
$$

Therefore, $\int^{T^{*}}\left\|\left(u^{n} \cdot \nabla\right) u^{n}\right\|_{L_{2}}^{4} \mathrm{~d} \tau \leq C T^{*}$ for all $n$; which, in turn, implies $\left(u^{n} \cdot \nabla\right) u^{n} \in L_{2}\left(0, T^{*}\right) \times L_{2}(\Omega)$. Now, let us show thal $u$ satisfies 1.5. Evidently, $u^{n}$ and $\rho^{n}$ satisfy 1.5 for

$$
\Phi=\sum_{k=1}^{m} H_{k}(t) \varphi_{k}, H_{k} \in C^{1}\left[0, T^{*}\right], H_{k}\left(T^{*}\right)=0 .
$$

Since $u^{n} \rightarrow u$ weakly in $L_{2}\left(0, T^{*}\right) \times J_{1}(\Omega)$, then

$$
\int_{0}^{T^{*}}\left\langle\nabla u^{n}, \nabla v\right\rangle \mathrm{d} x \mathrm{~d} t \underset{n \rightarrow \infty}{\rightarrow} \int_{0}^{T^{*}}\langle\nabla u, \nabla v\rangle \mathrm{d} x \mathrm{~d} t, v \in L_{2}\left(0, T^{*}\right) \times J_{1}(\Omega)
$$

Analogously, we have that $\int_{0}^{T^{*}}\left\langle u^{n}, v\right\rangle \mathrm{d} t \underset{n \rightarrow \infty}{\rightarrow} \int_{0}^{T^{*}}\langle u, v\rangle \mathrm{d} t, \int_{0}^{T^{*}}\left\langle\rho^{n} e_{3}, \Phi\right\rangle \mathrm{d} t \underset{n \rightarrow \infty}{\rightarrow} \int_{0}^{T^{*}}\left\langle\rho e_{3}, \Phi\right\rangle \mathrm{d}$. It remains to prove the limit 


$$
\int_{0}^{T^{*}}\left\langle u^{n},\left(u^{n} \cdot \nabla\right) \Phi\right\rangle \mathrm{d} t \underset{n \rightarrow \infty}{\rightarrow} \int_{0}^{T^{*}}\langle u,(u \cdot \nabla) \Phi\rangle \mathrm{d} t .
$$

To verify 1.43 , we note first that

$$
\left\langle u^{n},\left(u^{n} \cdot \nabla\right) \Phi\right\rangle-\langle u,(u \cdot \nabla) \Phi\rangle=\left\langle u^{n}-u,(u \cdot \nabla) \Phi\right\rangle+\left\langle u^{n},\left(\left(u^{n}-u\right) \cdot \nabla\right) \Phi\right\rangle .
$$

We integrate by $t$ the relation 1.44 and use the properties that $u_{n}$ is uniformly bounded in $L_{\infty}\left(0, T^{*}\right) \times J(\Omega)$ and that $u^{n} \rightarrow u$ strongly in $L_{2}\left[\left(0, T^{*}\right) \times(\Omega)\right]$. In this way, we obtain that 1.43 is valid. Now, we pass to the limit for $n \rightarrow \infty$ in

$$
\int_{0}^{T^{*}}\left\{\left\langle u^{n}, \Phi_{t}\right\rangle+\left\langle u^{n},\left(u^{n} \cdot \nabla\right) \Phi\right\rangle+v\left\langle\nabla u^{n}, \nabla \Phi\right\rangle+\left\langle\rho^{n} e_{3}, \Phi\right\rangle\right\} \mathrm{d} t+\left\langle u_{0}, \Phi(0)\right\rangle=0,
$$

and thus obtain

$$
\int_{0}^{T^{*}}\left\{\left\langle u, \Phi_{t}\right\rangle+\langle u,(u \cdot \nabla) \Phi\rangle+v\langle\nabla u, \nabla \Phi\rangle+\left\langle\rho e_{3}, \Phi\right\rangle\right\} \mathrm{d} t+\left\langle u_{0}, \Phi(0)\right\rangle=0
$$

for the functions $\Phi$ from 1.42. Due to the density of the set 1.42 , we have that 1.45 is valid for all the functions $\Phi$ from Definition 1, which completes the proof.

\section{Uniqueness of the Solutions}

\section{Theorem 2.}

The weak solution in sense of Definition 1, is unique.

Proof.

Let us exchange $\rho$ by $\frac{1}{N} \rho$ and thus rewrite the system 1.2 in a more symmetrical way:

$$
\begin{aligned}
& \frac{\partial}{\partial t}\left(\begin{array}{l}
u_{1} \\
u_{2} \\
u_{3}
\end{array}\right)-v\left(\begin{array}{l}
\Delta u_{1} \\
\Delta u_{2} \\
\Delta u_{3}
\end{array}\right)+\left(\begin{array}{l}
\frac{\partial p}{\partial x_{1}} \\
\frac{\partial p}{\partial x_{2}} \\
\frac{\partial p}{\partial x_{3}}
\end{array}\right)=\left(\begin{array}{c}
0 \\
0 \\
-\frac{1}{N} \rho
\end{array}\right)-\left(\begin{array}{l}
u \cdot \nabla u_{1} \\
u \cdot \nabla u_{2} \\
u \cdot \nabla u_{3}
\end{array}\right), \\
& \frac{\partial \rho}{\partial t}-\frac{1}{N} u_{3}=0, \frac{\partial u_{1}}{\partial x_{1}}+\frac{\partial u_{2}}{\partial x_{2}}+\frac{\partial u_{3}}{\partial x_{3}}=0 .
\end{aligned}
$$

Let $\left(u, \rho_{1}, p_{1}\right)$ and $\left(w, \rho_{2}, p_{2}\right)$ be two solutions of $2.1-2.2$ which satisfy the conditions $1.3-1.4$ and also the conditions of Theorem 1 . We denote $U=w-u, P=p_{2}-p_{1}$, and thus obtain

$$
\frac{\partial U}{\partial t}-v \Delta U=-\nabla P+\left(\begin{array}{c}
0 \\
0 \\
-\frac{1}{N}\left(\rho_{2}-\rho_{1}\right)
\end{array}\right)+\left(\begin{array}{l}
u \cdot \nabla u_{1}-w \cdot \nabla w_{1} \\
u \cdot \nabla u_{2}-w \cdot \nabla w_{2} \\
u \cdot \nabla u_{3}-w \cdot \nabla w_{3}
\end{array}\right) .
$$

From 2.2 we have $\rho_{2}-\rho_{1}=\frac{1}{N} \int_{0}^{t} U_{3}(s, x)$ ds. We observe that we can express 2.3 as follows: 


$$
\frac{\partial U}{\partial t}-v \Delta U=-\nabla P+\left(\begin{array}{c}
0 \\
0 \\
-\frac{1}{N}\left(\rho_{2}-\rho_{1}\right)
\end{array}\right)+\left(\begin{array}{l}
u \cdot \nabla U_{1}-U \cdot \nabla w_{1} \\
u \cdot \nabla U_{2}-U \cdot \nabla w_{2} \\
u \cdot \nabla U_{3}-U \cdot \nabla w_{3}
\end{array}\right) .
$$

We multiply 2.4 by $U$, integrate by parts in $\Omega$. In this way, we have

$$
\frac{1}{2} \frac{\mathrm{d}}{\mathrm{d} t}\|U\|^{2}+v\|\nabla U\|^{2}=-\frac{1}{N^{2}}\left\langle\int_{0}^{t} U_{3}(s, x) \mathrm{d} s, U_{3}\right\rangle-\langle(U \cdot \nabla) w, U\rangle .
$$

By using the generalized Hölder inequality and Young inequality, together with the inequity $\|U\|_{L_{4}} \leq C\|U\|^{\frac{1}{4}}\|\nabla U\|^{\frac{3}{4}}$, we can estimate the last term in 2.5 as

$$
|\langle(U \cdot \nabla) w, U\rangle| \leq\|U\|_{L_{4}}^{2}\|\nabla w\| \leq C\|U\|^{\frac{1}{2}}\|\nabla U\|^{\frac{3}{2}}\|\nabla w\| \leq \varepsilon C\|\nabla U\|^{2}+C_{1}(\varepsilon)\|U\|^{2}\|\nabla w\|^{4} .
$$

If we take $\varepsilon=\frac{v}{C}$, then we will have the estimate

$$
\frac{1}{2} \frac{\mathrm{d}}{\mathrm{d} t}\|U\|^{2} \leq \frac{1}{N^{2}}\left|\left\langle\int_{0}^{t} U_{3}(s, x) \mathrm{d} s, U_{3}\right\rangle\right|+C_{1}\|U\|^{2}\|\nabla w\|^{4} .
$$

Now we would like to estimate the term $\mid\left\langle\int_{0}^{t} U_{3}(s, x) \mathrm{d} s, U_{3}\right\rangle$. It is easy to see that

$$
\begin{aligned}
\left.\| \int_{0}^{t} U_{3}(s, x) \mathrm{d} s, U_{3}\right\rangle \mid & \leq \iint_{\Omega} \int_{0}^{t} U_{3}(s, x) \mathrm{d} s|| U_{3}(t, x) \mid \mathrm{d} x \leq \frac{1}{2}\left(\int_{\Omega}\left(\int_{0}^{t} U_{3}(s, x) \mathrm{d} s\right)^{2} \mathrm{~d} x+\int_{\Omega} U_{3}^{2}(t, x) \mathrm{d} x\right) \\
& \leq \frac{1}{2}\left(t \int_{0}^{t} \int_{\Omega} U_{3}^{2}(s, x) \mathrm{d} x \mathrm{~d} s+\int_{\Omega} U_{3}^{2}(t, x) \mathrm{d} x\right) \leq \frac{t}{2} \int_{0}^{t}\|U\|^{2} \mathrm{~d} s+\frac{1}{2}\|U\|^{2} .
\end{aligned}
$$

In this way, from 2.7 we obtain the estimate

$$
\frac{\mathrm{d}}{\mathrm{d} t}\|U\|^{2} \leq \frac{1}{N^{2}} t \int_{0}^{t}\|U\|^{2} \mathrm{~d} s+\left(1+C\|\nabla w\|^{4}\right)\|U\|^{2} .
$$

Now, let us consider the following initial value problem for the function $y(t)$ :

$$
\left\{\begin{array}{l}
y^{\prime}=f(t, y)=\phi(t) y+\frac{t}{N^{2}} \int_{0}^{t} y(s) \mathrm{d} s \\
y(0)=0 .
\end{array}\right.
$$

Evidently, for continuous $\phi(t)$ the unique solution of the problem 2.9 is $y \equiv 0$.

By comparison principle, therefore, for every solution of the differential inequality

$$
\left\{\begin{array}{l}
z^{\prime} \leq f(t, z) \\
z(0) \leq 0
\end{array}\right.
$$

the property holds: $z(t) \leq 0$. In this way, we conclude from 2.9 that $\|U\|^{2}=0$, which implies that $u=w$.

Using 2.2 we obtain also that $\rho_{2}=\rho_{1}$, and thus the theorem is proved.

\section{Acknowledgements}

This research was partially supported by “Fondo de Investigaciones Facultad de Ciencias-Uniandes”. 


\section{References}

[1] Cushman-Roisin, B. and Beckers, J. (2011) Introduction to Geophysical Fluid Dynamics. Academy Press, New York.

[2] Tritton, D. (1990) Physical Fluid Dynamics. Oxford UP, Oxford.

[3] Kundu, P. (1990) Fluid Mechanics. Academy Press, New York.

[4] Landau, L. and Lifschitz, E. (1959) Fluid Mechanics. Pergamon Press, New York.

[5] Makarenko, N., Maltseva, J. and Kazakov, A. (2009) Conjugate Flows and Amplitude Bounds for Internal Solitary Waves. Nonlinear Processes in Geophysics, 16, 169-178. http://dx.doi.org/10.5194/npg-16-169-2009

[6] Maurer, B., Bolster, D. and Linden, P. (2010) Intrusive Gravity Currents between Two Stably Stratified Fluids. Journal of Fluid Mechanics, 647, 53-69. http://dx.doi.org/10.1017/S0022112009993752

[7] Birman, V. and Meiburg, E. (2007) On Gravity Currents in Stratified Ambients. Physics of Fluids, 19, $602-612$. http://dx.doi.org/10.1063/1.2756553

[8] Maslennikova, V. and Giniatoulline, A. (1992) On the Intrusion Problem in a Viscous Stratified Fluid for Three Space Variables. Mathematical Notes, 51, 374-379. http://dx.doi.org/10.1007/BF01250548

[9] Giniatoulline, A. and Zapata, O. (2007) On Some Qualitative Properties of Stratified Flows. Contemporary Mathematics, Series AMS, 434, 193-204.

[10] Giniatoulline, A. and Castro, T. (2012) On the Spectrum of Operators of Inner Waves in a Viscous Compressible Stratified Fluid. Journal of the Faculty of Science, University of Tokyo, 19, 313-323.

[11] Heywood, J. (1980) The Navier-Stokes Equations: On the Existence, Regularity and Decay of Solutions. Indiana University Mathematics Journal, 29, 639-681. http://dx.doi.org/10.1512/iumj.1980.29.29048

[12] Tartar, L. (2006) An Introduction to Navier-Stokes Equations and Oceanography. Springer, Berlin.

[13] Temam, R. (2000) Navier-Stokes Equations: Theory and Numerical Analysis. AMS Chelsea Publishing, New York.

[14] Sohr, H. (2012) The Navier-Stokes Equations: An Elementary Functional Analytic Approach. Birkhäuser, Zurich.

[15] Boyer, F. (2005) Elements d'analyse pour l'etude de quelques modeles d'ecoulements de fluides visqueux incompressibles. Springer, Berlin. 